Memorias del 1 Congreso Nacional de Investigación en Enseñanza de la Biología.

VI Encuentro Nacional de Investigación en Enseñanza de la Biología y la Educación Ambiental. ISSN 2027 1034. P. p. 133-140.

\title{
CONCEPCIONES SOBRE PRÁCTICAS DE CAMPO DE FUTUROS PROFESORES DE BIOLOGÍA
}

\section{FUTURE BIOLOGY TEACHERS' CONCEPTIONS ABOUT FIELD PRACTICES}

\author{
POR: Elías Francisco Amórtegui Cedeño ${ }^{1}$ \\ Édgar Orlay Valbuena Ussa ${ }^{2}$
}

\section{Resumen}

Presentamos resultados parciales de la investigación "Concepciones sobre Prácticas de Campo y su relación con la construcción del Conocimiento Profesional del Profesor, de futuros docentes de Biología de la Universidad Pedagógica Nacional (Colombia)". El estudio se llevó a cabo durante seis meses con un grupo de 23 futuros profesores. Consistió en la aplicación de cuestionarios, talleres, entrevistas y observación participante. Para este caso, nos referimos particularmente a las categorías: Enfoque Didáctico y Aspectos relacionados con la Formación Docente. Los hallazgos muestran que la mayoría de los futuros maestros conciben la Práctica de Campo y su evaluación desde un enfoque constructivista, y en algunos aún predominan enfoques tradicionales. Con relación a la formación docente, identifican los Componentes y Fuentes del Conocimiento Profesional del Profesor.

Palabras clave: Concepción, Práctica de campo, Conocimiento Profesional del Profesor, Enseñanza de la Biología.

\section{Abstract}

We present research partial results of Future Biology teachers from Universidad Pedagógica Nacional (Colombia) Conceptions about field practices and their relationship with Teacher's Professional Knowledge construction". The study was carried out during six months with a group of 23 future teachers. It was based on the questionnaires, workshops, interviews and participant observations application. In this case, we particularly refer to didactic approach and aspects linked to teacher's education category. The discoveries show that most of the future teachers conceive field practice and its evaluation from a constructive approach and in some of them traditional approaches predominate. In relation to teacher's

${ }^{1}$ folkerpan@hotmail.com. Universidad Pedagógica Nacional. Departamento de Biología. Grupo de Investigación Conocimiento Profesional del Profesor de Ciencias.

²edgarorlay@hotmail.com valbuena@pedagogica.edu.co Universidad Pedagógica Nacional. Departamento de Biología. Grupo de Investigación Conocimiento Profesional del Profesor de Ciencias. 
Memorias del I Congreso Nacional de Investigación en Enseñanza de la Biología. VI Encuentro Nacional de Investigación en Enseñanza de la Biología y la Educación Ambiental. ISSN 2027 1034. P. p. 133-140.

education components and teacher's professional knowledge Sources are identified.

Keywords: conception, field work, teacher's professional knowledge, Biology teaching.

\section{INTRODUCCIÓN}

De la misma manera como profesionales de la medicina, $u$ otros campos, tienen un conocimiento particular sobre su disciplina, los profesores cuentan con un conocimiento particular. De acuerdo a Tardif (2004), el trabajo docente se diferencia de otros trabajos ya que:

- Su objeto es heterogéneo y está conformado por sujetos humanos e interacciones humanas.

- Sus objetivos son ambiguos, amplios, ambiciosos y a largo plazo.

- Implica relaciones complejas.

- Su producto es intangible e inmaterial, ya que el proceso de aprender a ser maestro es simultáneo con la producción (enseñar, es decir, hacer aprender), con lo cual es difícil separar al trabajador del resultado. Es decir que es difícil observar al profesor fuera de su lugar de producción.

Teniendo presente la complejidad, tanto conceptual como contextual de la enseñanza, para el caso del Conocimiento Profesional del Profesor es difícil identificar los componentes que lo integran y su estructura. Las investigaciones fundamentalmente diferencian como componentes cuatro grandes componentes (Valbuena, 2007):

- El conocimiento de los contenidos, del objeto o materia de enseñanza, que en este trabajo se denominará conocimiento del contenido disciplinar.

- El conocimiento pedagógico.

- El conocimiento necesario para enseñar un saber en particular, denominado Conocimiento Didáctico del Contenido (CDC).

- El conocimiento del contexto.

Desde la perspectiva del Conocimiento Profesional del Profesor, las Prácticas de Campo y en general, los Trabajos Prácticos, son considerados estrategias de enseñanza, y desde esta perspectiva son un componente fundamental en el Conocimiento Didáctico del Contenido (Valbuena, 2007), sin embargo las investigaciones sobre el Conocimiento del Profesional del Profesor, para el caso de Biología, son escasos y en su mayoría se han realizado respecto al conocimiento disciplinar que se enseña (Subject matter Knowledge). 
Memorias del 1 Congreso Nacional de Investigación en Enseñanza de la Biología.

VI Encuentro Nacional de Investigación en Enseñanza de la Biología y la Educación Ambiental. ISSN 2027 1034. P. p. 133-140.

El Trabajo Práctico y la formación del profesorado en la enseñanza de las Ciencias han venido constituyéndose en una faceta muy valorada por los investigadores (Rodrigo et al, 1999). Específicamente las Prácticas de Campo (PC) se consideran importantes en la formación inicial de profesores de Ciencias (Dourado, 2006), al concebirse como una estrategia de enseñanza que permite relacionar la teoría con la práctica y favorecer el aprendizaje de diversos contenidos, constituyendo además una fuente de información directa, ejemplos y experiencias.

Dado que el objeto de esta investigación está relacionado con las concepciones que tienen futuros docentes sobre Prácticas de Campo, es fundamental establecer su importancia en el marco del Conocimiento Profesional del Profesor.

De acuerdo a Porlán y Rivero (1998), dentro del Conocimiento del Profesor las concepciones se refieren tanto a saberes académicos como a saberes experienciales. Los primeros son aquellos que tienen los profesores que pueden estar relacionados con el currículo o las Ciencias de la Educación, fundamentalmente generados en la formación inicial, de allí la importancia de analizar los procesos formativos de los futuros docentes que hacen parte de esta investigación; los segundos saberes son de naturaleza explícita y organizada, relacionados con los procesos de enseñanza-aprendizaje (aprendizaje de los alumnos, metodología, evaluación, fines educativos, entre otros).

Porlán, Rivero y Martín (1997), realizan una revisión acerca de las investigaciones sobre las concepciones en el marco del Conocimiento del Profesor. Estos autores plantean como primera tendencia en las investigaciones, una Perspectiva constructivista, en la cual las concepciones son consideradas como "herramientas" para poder interpretar la realidad y conducirse a través de ella y "barreras" que impiden adoptar perspectivas y cursos de acción diferentes. Las concepciones pueden evolucionar a través de un proceso de restructuración que puede o no ser consciente, basado en la interacción con otras ideas y experiencias de los sujetos, de allí la importancia de la discusión y socialización que se llevó a cabo durante el desarrollo del taller en el presente estudio.

Dicha evolución puede favorecerse y acelerarse con base en procesos de investigación dirigidos, seleccionando problemas relevantes, favoreciendo la toma de conciencia de ideas buscando el contraste riguroso con otros puntos de vista, con otras formas de actuar y tomando decisiones que han surgido de diversos procesos de reflexión. 
Memorias del 1 Congreso Nacional de Investigación en Enseñanza de la Biología. VI Encuentro Nacional de Investigación en Enseñanza de la Biología y la

Educación Ambiental. ISSN 2027 1034. P. P. 133-140.

De acuerdo con lo anterior, es importante analizar el papel de las PC en los programas curriculares de formación de docentes en Ciencias. Sin embargo, es de destacar que existen pocas investigaciones sobre la incidencia de dichas actividades en la formación de profesores de Biología y mucho menos sobre su aporte a la construcción del Conocimiento Profesional del Profesor (Amórtegui y Valbuena, 2010).

\section{METODOLOGÍA}

El estudio se llevó a cabo desde un enfoque cualitativo, empleando el método de análisis de contenido en el proceso de sistematización, y usando como herramientas de recolección de información el taller, la entrevista, la observación participante y el cuestionario (el cual diseñamos y validamos con pares expertos). El grupo objeto de estudio consistió en 23 estudiantes entre 17 y 25 años de edad, pertenecientes en su mayoría a los estratos socioeconómicos 2 y 3 . Las categorías de la investigación corresponden a lo planteado por Amórtegui y Correa (2009), y Puntes y Valbuena (2011), (Ver Tabla 1).

\begin{tabular}{|c|c|}
\hline Categoría & Subcategorías \\
\hline \multirow{3}{*}{ NATURALEZA } & Imagen de práctica \\
\hline & Relación teoría- práctica \\
\hline & Clase de Trabajo Práctico \\
\hline \multicolumn{2}{|l|}{ FINALIDADES } \\
\hline \multirow[t]{4}{*}{ METODOLOGÍA } & Estructura de la Guía de Campo \\
\hline & Enfoque didáctico \\
\hline & Momento de realización \\
\hline & Roles \\
\hline \multirow[t]{3}{*}{ EVALUACIÓN } & Tipos \\
\hline & Roles \\
\hline & Contenidos a evaluar \\
\hline \multirow{4}{*}{$\begin{array}{lrl}\text { RELACIÓN CON } & \text { ASPECTOS } \\
\text { EPISTEMOLÓGICOS } & \end{array}$} & Biología como Ciencia \\
\hline & Objeto de estudio de la Biología \\
\hline & Producción de conocimiento biológico \\
\hline & Imagen de Ciencia \\
\hline \multicolumn{2}{|l|}{$\begin{array}{l}\text { ASPECTOS RELACIONADOS CON } \\
\text { LA FORMACIÓN DOCENTE }\end{array}$} \\
\hline $\begin{array}{l}\text { ASPECTOS RELACIONADOS CON } \\
\text { EL CURRÍCULO DEL PCLB }\end{array}$ & \\
\hline
\end{tabular}

Tabla 1. Categorías de la investigación (Tomado de Amórtegui y Correa, 2009; Puentes y Valbuena, 2011). 
Memorias del I Congreso Nacional de Investigación en Enseñanza de la Biología. VI Encuentro Nacional de Investigación en Enseñanza de la Biología y la Educación Ambiental. ISSN 2027 1034. P. P. 133-140.

\section{RESULTADOS Y DISCUSIÓN}

A continuación presentamos los hallazgos para dos de las categorías de investigación. En algunos casos, ilustramos con declaraciones textuales de los futuros profesores de Biología (destacamos la información relevante subrayando).

Con relación al Enfoque Didáctico, entendido como la relación entre la PC y un referente teórico didáctico desde la perspectiva de la enseñanza-aprendizaje de las ciencias y la Biología, 18 futuros profesores al comienzo del proceso formativo se identifican con el Constructivismo, ya que tienen en cuenta aspectos como el interés, los puntos de vista, las ideas previas, el proceso de aprendizaje y la experiencia de los alumnos. Además, consideran que el docente es un guía, mientras que el estudiante es un constructor de conocimiento que pregunta, refuerza y complementa lo que aprende, siendo la Salida de Campo una actividad retroalimentada tanto por docentes como por estudiantes.

E10.Cl.6: [Refiriéndose al momento de realización de una SC] "Durante el desarrollo de los temas porque los estudiantes llevan una serie de ideas previas, preconceptos que en la salida pueden reafirmarse o reformularse."

Cabe aclarar, que de los 18 estudiantes, 9 mantuvieron esta concepción al final del proceso formativo.

Con relación al Conocimiento Profesional del Profesor y la construcción del Conocimiento Didáctico del Contenido Biológico, es fundamental que los docentes conciban una enseñanza de la Biología que persiga un aprendizaje significativo y el desarrollo de capacidades metacognitivas en los alumnos, teniendo en cuenta una enseñanza que contempla muy especialmente las ideas de los alumnos; por ello se realizan actividades para su detección, activación, confrontación y reestructuración.

Por otra parte, 4 estudiantes-profesores se identificaron con un Enfoque Tradicional, ya que el docente se encarga de la orientación de la actividad, mientras que el alumno se limita exclusivamente a la aplicación de su conocimiento, la observación y la experimentación en la Salida de Campo.

E11.CF.6: "El docente sería el guía de la salida pues apoyaría el trabajo de campo y daría las instrucciones para desarrollarlo 
Memorias del I Congreso Nacional de Investigación en Enseñanza de la Biología.

VI Encuentro Nacional de Investigación en Enseñanza de la Biología y la

Educación Ambiental. ISSN 2027 1034. P. p. 133-140.

adecuadamente. Los estudiantes serían quienes refuercen los conocimientos desarrollando el trabajo de campo."

Con relación a la construcción del Conocimiento Profesional del Profesor y el Conocimiento Didáctico del Contenido Biológico, Valbuena (2007) reconoce la importancia de superar e ir más allá de los enfoques tradicionales de la enseñanza de la Biología en los que prima la explicación exclusiva de contenidos biológicos, fundamentalmente conceptuales, tal y como están establecidos en los libros de texto y en los programas curriculares donde los protagonistas de la enseñanza son el profesor y los contenidos, generando así que todas las decisiones en el aula sean tomadas por el docente.

En cuanto a los Aspectos relacionados con la formación docente, 10 estudiantes identifican tanto al comienzo como al final del proceso formativo los componentes y fuentes del Conocimiento Profesional del Profesor, ya que para ellos las SC enriquecen los ámbitos biológico, humanístico, pedagógico, social, político, educativo, al igual que la experiencia.

E17.Cl.5: [Refiriéndose al aporte de la SC a la formación docente] "El reconocimiento de diversos contextos, organismos, situaciones, además del acercamiento con lo que se considera la práctica de lo biológico y pedagógico, permitiendo establecer que la generalidad sesga lo especial y particular de un organismo, comunidad, ecosistema etc."

Es fundamental que los futuros profesores identifiquen por una parte la importancia de la experiencia como una fuente de construcción del Conocimiento Profesional del Profesor (Porlán y Rivero, 1998; Tardif, 2004; Valbuena, 2007) y por otra, que éste consta de diversos componentes de tal forma que no basta con que el profesor domine académicamente el conocimiento disciplinar específico que se enseña para que sea un buen profesor, en donde la organización y secuenciación del currículo obedece única y exclusivamente a la lógica de la ciencia. Los planteamientos de estos futuros profesores implican pensar que se requiere así, de un conocimiento pedagógico y didáctico.

\section{CONCLUSIONES}

Los futuros profesores conciben las Prácticas de Campo desde una perspectiva constructivista en su gran mayoría; sin embargo, siguen estando presentes ideas sobre la enseñanza tradicional en los maestros en formación. 
Memorias del I Congreso Nacional de Investigación en Enseñanza de la Biología.

VI Encuentro Nacional de Investigación en Enseñanza de la Biología y la Educación Ambiental. ISSN 2027 1034. P. p. 133-140.

Sobre el Conocimiento Profesional del Profesor, y la estructuración del Conocimiento Didáctico del Contenido, es favorable reconocer que los futuros profesores de Biología identifican dichos conocimientos y sus componentes a través del aporte de las Prácticas de Campo a su formación.

Con relación a la naturaleza de las concepciones, durante el proceso formativo pudo evidenciarse: que perduran durante los procesos académicos, son resistentes al cambio en su gran mayoría y en algunos casos contradictorias. Sin embargo, para ciertos estudiantes existió una modificación en sus concepciones, probablemente debido a las actividades de discusión, confrontación, debate y explicitación, tanto a nivel individual como grupal, durante el proceso formativo, favorecido por los talleres, entrevistas y el abordaje de las Prácticas de Campo como contenido formativo en los espacios académicos que cursan los futuros profesores de Biología.

\section{BIBLIOGRAFÍA}

- Amórtegui, E; Gutiérrez, A; Medellín, F (2009). Las prácticas de campo en la construcción del conocimiento profesional de futuros profesores de Biología. En Bio-grafía, escritos sobre la biología y su enseñanza. 2 (1).En: http://www.pedagogica.edu.co/revistas/ojs/index.php/biografia/article/viewFil e/160/129.

- Amórtegui, E Y Correa, M (2009). Las Prácticas de Campo Planificadas en el Proyecto Curricular de Licenciatura en Biología de la Universidad Pedagógica Nacional. Caracterización desde la perspectiva del Conocimiento Profesional del Profesor de Biología. Tesis para optar al título de Licenciado en Biología. Universidad Pedagógica Nacional.

- Amórtegui Elías y Valbuena Ussa Edgar (2010). Prácticas de campo en la enseñanza de la Biología y la formación docente: una revisión de antecedentes. IX Jornadas nacionales y IV Congreso internacional de enseñanza de la Biología. San Miguel de Tucumán 9 de octubre.

- Dourado, L (2006). Concepçoes e práticas dos profesores de Ciencias Naturais Relativas à implementaçao Integrada do Trabalho Laboratorial e do Trabalho de Campo. Revista Electrónica de Enseñanza de las Ciencias. 5 (1). Pp. 192-212.

- Porlán R; Rivero, A y Martín, R (1997). Conocimiento profesional y epistemología de los profesores I: Teoría, métodos e instrumentos. Enseñanza de las Ciencias. 15 (2) 155-171.

- Porlán, R. Y Rivero, A. (1998). El conocimiento de los profesores: una propuesta formativa en el área de ciencias. Sevilla: Díada. 
Memorias del 1 Congreso Nacional de Investigación en Enseñanza de la Biología. VI Encuentro Nacional de Investigación en Enseñanza de la Biología y la Educación Ambiental. ISSN 2027 1034. P. p. 133-140.

- Puentes, M. y Valbuena, E. (2011). Sistema de categorías para análisis didáctico de trabajos prácticos en la enseñanza de la Biología. Bio-grafía. Escritos sobre la Biología y su Enseñanza. (en prensa).

- Rodrigo, M; Morcillo, J; Borges, R; Calvo, M; Cordeiro, N; García, F; Raviolo, A (1999) Concepciones sobre el Trabajo Práctico de campo (TPc): una aproximación al pensamiento de los futuros profesores. Revista Complutense de Madrid. 10 (2), 261-285.

- Tardif, M. (2004). Los saberes del docente y su desarrollo profesional. Traducción de Pablo Manzano. Madrid: Narcea.

- Valbuena, E. (2007). El Conocimiento Didáctico del Contenido Biológico. Estudio de las concepciones disciplinares y didácticas de futuros docentes de la Universidad Pedagógica Nacional (Colombia). Tesis para optar al título de Doctor en Didáctica de las Ciencias Experimentales. Universidad Complutense de Madrid. 